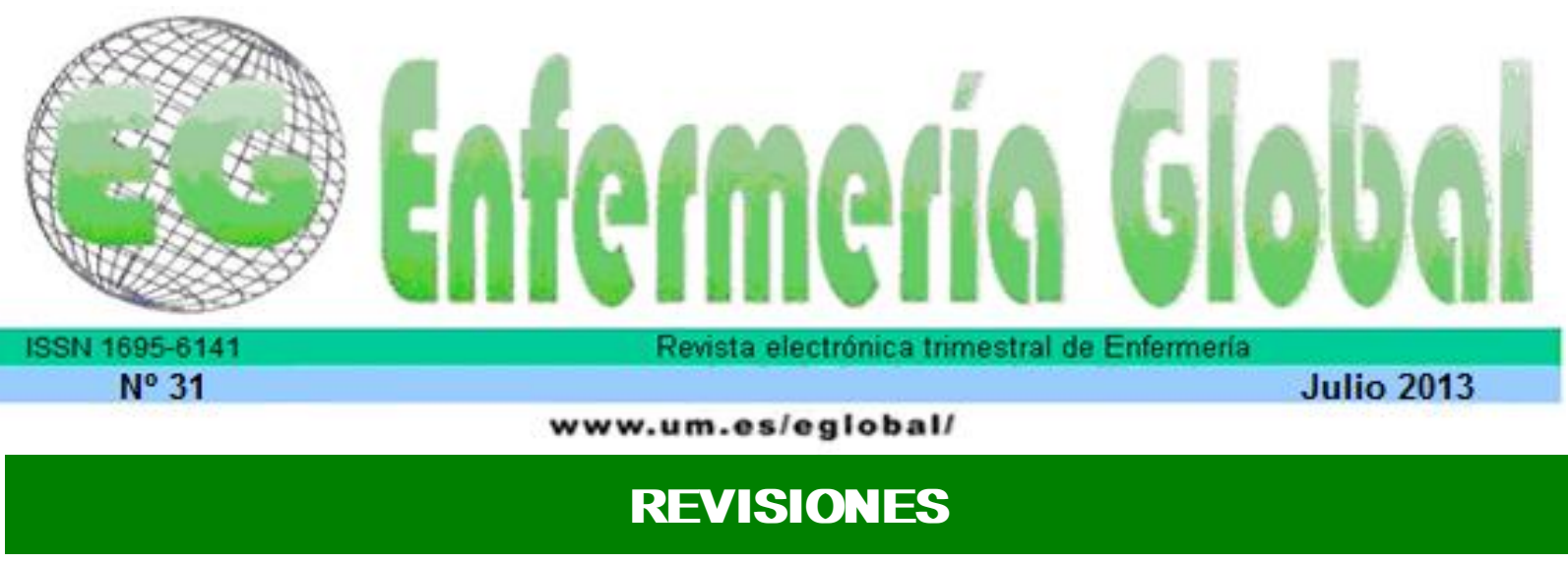

\title{
Cuidados de enfermería en situación de cárcel según Waldow: entre lo profesional y lo expresivo
}

Cuidados de enfermagem em situação de cárcere segundo Waldow: entre o profissional e o expressivo

Nursing care in prison by Waldow: between the profesional and the affectionate

\section{*Souza Santos, Fernanda ${ }^{* *}$ Cavalcanti Valente, Geilsa Soraia ${ }^{* * *}$ Souza, Lídia Marina do Carmo ${ }^{* * * *}$ Caldeira dos Santos, Mauro Leonardo Salvador ***** Silva dos Santos, Ivanlésio ******Schwartz, Maria da Penha}

*Mestranda en Enfermería Asistencial por la Escuela de Enfermería Aurora de Afonso Costa EEAAC/UFF. **Doctora en Enfermería; Profesora Adjunta del Departamento de Fundamentos de Enfermería y Administración de la EEAAC/UFF E mail: geilsavalente@yahoo.com.br ***Enfermera, Postgraduada en Docencia de Enseñanza Superior, Docente en Enfermería de la Universidad del Estado de Rio de Janeiro. ${ }^{* * * *}$ Doctor en Enfermería por la Universidad Federal de Santa Catarina. Profesor del Departamento de Enfermería Médico-Quirúrgica de la Universidad Federal Fluminense.

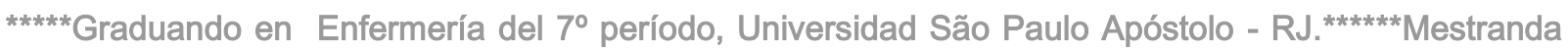
Enfermería Profesional EEAAC-UFF. Brasil.

Palabras clave: Cuidados de Enfermería; Enfermería psiquiátrica, Prisiones

Palavras-chave: Cuidados de enfermagem; Enfermagem psiquiátrica; Prisões

Keywords: Nursing; Psychiatric Nursing; Prisons.

\section{RESUMEN}

Los cuidados de enfermería se pueden desarrollar en diferentes espacios, como en el Sistema Penal de Prisiones. Esta es una revisión de la literatura con análisis cualitativo de la información, realizada con artículos científicos, localizados en periódicos en la Biblioteca Virtual en Salud (BVS), en las bases de banco de datos (BDENF), Literatura Latino Americana y del Caribe en Ciencias de la Salud (LILACS) y Scientific Electronic Library Online (SCIELO), publicados entre los años 2000 a 2010 utilizando las palabras clave: enfermería, enfermería psiquiátrica y prisiones, dando como resultado el hallazgo de 04 artículos, con texto completo en portugués, lo que contribuyó a la elaboración de 03 categorías, en orden de los estudios de Waldow sobre el cuidado humano.

El objetivo era levantar junto a la producción científica brasileña de enfermería los cuidados de enfermería administrados al interno en la cárcel. 
El análisis mostró que el $75 \%$ de los textos se refería a los Cuidados Profesionales y el $25 \%$ a los Cuidados Expresivos como acciones de enfermería.

En conclusión, la enfermería, al utilizar los estímulos físicos y mentales, busca hacer que el sujeto de cuidados se sienta apoyado y valorado en sus necesidades.

\section{RESUMO}

O cuidado de enfermagem pode se desenvolver em vários espaços, como nas Unidades do Sistema Penal. Trata-se de revisão bibliográfica, com análise qualitativa de informações, realizada com artigos científicos, localizados em periódicos na biblioteca Virtual em Saúde (BVS), nas bases Banco de Dados da Enfermagem (BDENF), Literatura Latino-Americana e do Caribe em Ciências da Saúde (LILACS) e Scientific Electronic Library Online (SCIELO), publicados entre os anos de 2000 a 2010, utilizando os descritores: cuidados de enfermagem, enfermagem psiquiátrica e prisões, resultando no achado de 04 artigos, com texto completo em português, que contribuíram para a elaboração de 03 categorias de desenvolvimento, classificadas a partir dos estudos de Waldow sobre o cuidado humano.

O objetivo foi levantar junto à produção científica brasileira de enfermagem os cuidados de enfermagem administrados ao interno em situação de cárcere.

A discussão mostrou que $75 \%$ dos textos referiram os Cuidados Profissionais e $25 \%$ os Cuidados Expressivos como ações de enfermagem.

Concluindo que a enfermagem, ao utilizar os estímulos físico-mentais, busca fazer o sujeito de cuidados, sentir-se apoiado e valorizado em suas necessidades.

\section{ABSTRACT}

Nursing care can develop in various areas, such as the Units of the Penal System. This is a literature review, qualitative analysis of information held with papers, periodicals located in the Virtual Health Library (VHL), the bases Database of Nursing (BDENF), Latin American and Caribbean Health Sciences Health (LILACS) and Scientific Electronic Library Online (SciELO), published between the years 2000-2010, using the descriptors: nursing, psychiatric nursing and prisons, resulting in the finding of 04 articles with full text in Portuguese, which contributed to the development of 03 developed categories, classified from Waldow studies on human care.

The objective was to raise with the Brazilian scientific production in nursing care administered to the internal situation in prison.

The discussion showed that $75 \%$ of the texts mentioned the Care Professionals and Care $25 \%$ Expressive as nursing actions.

To conclude, nursing by means of using physical-mental stimuli, seeks to make the subject of care feel supported and valued in their needs.

\section{INTRODUCCIÓN}

El cuidado, siendo inherente al ser humano, se dispone de forma espontánea y consiste en el respeto a la dignidad humana, y en la sensibilidad para con el sufrimiento ajeno, en la ayuda para enfrentarlo, superarlo y hasta incluso en la preparación emocional de aceptación de lo inevitable. Este proceso incluye crecimiento y actualización ${ }^{(1-2)}$.

La expresión "cuidar" viene del latín, y significa "cura" (coera), es una acción que revela una actitud de desvelo y de preocupación por el otro, o sea, la disponibilidad del yo, enfocando al otro como sujeto de atenciones ${ }^{(3)}$. Parece que la filología de la 
palabra "cuidado" indica que cuidar es más que un acto singular; es un modo de ser, la forma como la persona se estructura y se realiza en el mundo de los otros ${ }^{(4)}$.

En la enfermería, el cuidar parte de la misma génesis, pues lo sitúa como compañero de la sociedad, que respetando la dignidad y la individualidad del cliente/paciente, invierte en la promoción, manutención, adquisición y/o recuperación del estado de equilibrio físico, mental y social, con acciones dirigidas al ser humano como un todo, sin fragmentar su todo biológico, psicológico y socio-cultural, en acciones que consideran el respeto a las ideas, a las opiniones individuales y $\operatorname{colectivas}^{(5)}$.

Así, se percibe que los cuidados que la enfermería otrora daba a la sociedad, tuvieron que adecuarse a las necesidades actuales y hoy el trabajo de enfermería se da en varios escenarios y espacios, desempeñando acciones preventivas y cuidados para la clientela, bastante diversificada, como en las comunidades, domicilios, algunas instituciones de enseñanza, plataformas y navíos de petróleo, así como en unidades del Sistema Penal, destinado a personas tuteladas por el Estado.

La atención a la salud de la población del sistema de prisiones, se encuentra inserta en el Sistema Único de Salud (SUS), a través de la Orden Interministerial ํㅜ․ 777, garantizando así que se haga efectivo el derecho ciudadano de las personas tuteladas por el Estado ${ }^{(6-7)}$. La creación por el Estado de instituciones penales tiene la finalidad de proteger a la población de aquellos que puedan representar algún tipo de amenaza o de peligro y uno de los objetivos de someter a alguien a una pena de privación de libertad es que, al ser retirados de la convivencia social, de alguna forma, el Sistema contribuye a que el interno al saldar su deuda con la sociedad adquiera condiciones de reinserción en ese contexto.

Para atender a tales expectativas, el sistema penal consta de diversas unidades prisiones como cárceles, hospitales-prisiones y manicomios judiciales, que tienen entre sí algunas funciones específicas y otras en común. Los criminales comunes, son los que cometieron delitos sin presentar trastornos mentales, cumplen pena en régimen cerrado, en unidad carcelaria común ${ }^{(8)}$. Los locos criminales tienen su situación para con la justicia, prevista en el artículo no 99 do Código Penal Brasileño, que reglamenta el Hospital de Custodia y Tratamiento Psiquiátrico (HCTP) para la recogida de aquellos que cometieran delito, derivado de trastorno mental ${ }^{(9)}$.

Las instituciones con fines de acogida son denominadas instituiciones totales, pues tienen carácter asilar y de encierro, por presentar toda una estructura que impide que el interno tenga contacto con el mundo externo. La institución total es un híbrido social, cuya ambigüedad se muestra en intensidad, estos lugares son parcialmente comunidad residencial y parcialmente organización formal ${ }^{(10-11)}$. Las cárceles, a pesar de ser lugares de detención, entre otros servicios contienen locales para la atención a la salud, son: ambulatorios, con consultorios destinados a la atención clínica; puestos - salas, equipadas para atender pequeñas intervenciones de emergencia, que excedan a los recursos asistenciales de la unidad prisional, son enviados al hospital general penal. Los hospitales-prisiones y entre ellos los manicomios judiciales, aunque sean lugares destinados al tratamiento de salud, añaden en su estructura, las características de prisión, simbolizadas por el sistema de seguridad, compuesto por equipo de guardia armada, altos muros con alambre, puertas de hierro, puertas cerradas con rejas, ventanas y candados $^{(11)}$. 


\section{OBJETIVO}

Con la intención de ampliar conocimientos sobre la temática de los cuidados de enfermería disponibilizados en el escenario de cárcel, se elaboró el siguiente objetivo de investigación: Levantar en la producción científica brasileña los cuidados de enfermería administrados al interno en situación de cárcel.

\section{MÉTODO}

La metodología utilizada fue la revisión de literatura con análisis cualitativo de informaciones. La investigación bibliográfica o de fuentes secundarias es la que se utiliza de datos colectados o extraídos a partir de toda bibliografia ya publicada ${ }^{(12)}$. A partir de ahí, se buscó por informaciones técnico-científicas en artículos publicados en periódicos de cuño científico. Toda investigación implica el levantamiento de datos de variadas fuentes, cualquiera que sean los métodos o técnicas empleadas ${ }^{(13)}$.

Así se recurrió a la busca sistematizada realizada en internet, por medio de la Biblioteca Virtual em Saúde (BVS) en las bases de datos LILACS, SCIELO y BDENF. Como criterio de inclusión, se optó por textos completos en portugués, el corte temporal fue de 08 años, comprendidos entre los años 2000 a 2008, utilizando los descriptores: cuidados de enfermería, enfermería psiquiátrica y prisiones. Lo que contribuyó al hallazgo de 04 artículos publicados en revistas de enfermería.

En el cuadro nำ, se encuentran los años de publicación, los nombres de los autores, los títulos y los lugaress donde se realizaron las investigaciones, así como los nombres de las revistas especializadas que publicaron los artículos: 
Cuadro1: Resultado de la búsqueda sistematizada

\begin{tabular}{|c|c|c|c|c|}
\hline Año & Autor & Título & Lugar & Publicaciones \\
\hline 2004 & Rosa, SRF & $\begin{array}{l}\text { Cuidar de Apenados: } \\
\text { percepção da equipe de } \\
\text { enfermagem. }\end{array}$ & $\begin{array}{l}\text { CTI de un } \\
\text { Hospital } \\
\text { General de } \\
\text { gran porte del } \\
\text { interior de Rio } \\
\text { Grande do Sul. }\end{array}$ & $\begin{array}{l}\text { Revista } \\
\text { Brasil }\end{array}$ \\
\hline 2006 & $\begin{array}{lr}\text { Rosinki, } & \text { TC. } \\
\text { Cordeiro, } & \text { CG. } \\
\text { Monticelli, } & \text { M. } \\
\text { Santos, EKA } & \end{array}$ & $\begin{array}{l}\text { Nascimento atrás das } \\
\text { grades: uma pratica de } \\
\text { cuidado direcionado a } \\
\text { gestantes, puérperas e } \\
\text { recém-nascidos em } \\
\text { privação de liberdade. }\end{array}$ & $\begin{array}{l}\text { Presidio } \\
\text { femenino, en } \\
\text { Santa Catarina. }\end{array}$ & $\begin{array}{l}\text { Revista Ciência, } \\
\text { Cuidado e Saúde }\end{array}$ \\
\hline 2006 & $\begin{array}{l}\text { Santos, MLS. } \\
\text { Souza, FS. } \\
\text { Santos, CVSC. }\end{array}$ & $\begin{array}{l}\text { As Marcas da Dupla } \\
\text { Exclusão: Experiências } \\
\text { da Enfermagem com o } \\
\text { Psicótico Infrator. }\end{array}$ & $\begin{array}{lr}\text { Hospital de } \\
\text { Custodia y } \\
\text { Tratamiento } \\
\text { Psiquiátrico en } \\
\text { RJ. }\end{array}$ & $\begin{array}{l}\text { Revista Texto e } \\
\text { Contexto }\end{array}$ \\
\hline 2008 & $\begin{array}{c}\text { Souza, MOS, } \\
\text { Passos, JP. }\end{array}$ & $\begin{array}{l}\text { A Prática de } \\
\text { Enfermagem no Sistema } \\
\text { Penal: Limites } \\
\text { Possibilidades. }\end{array}$ & $\begin{array}{lr}\text { Hospital } & \\
\text { general } & \text { del } \\
\text { Sistema } & \text { Penal } \\
\text { en RJ. } & \\
\end{array}$ & $\begin{array}{l}\text { Revista de Escola } \\
\text { de Enfermagem } \\
\text { Ana Nery }\end{array}$ \\
\hline
\end{tabular}

Con el material bibliográfico, se realizó la lectura exploratoria de todo el contenido; después, se procedió a la lectura selectiva. Los criterios de la lectura selectiva son los propósitos del trabajo, el problema formulado, las preguntas elaboradas, cuándo se cuestionó el asunto o, en otros términos, los objetivos intrínsecos del trabajo ${ }^{(14)}$.

Con la inteción de atender al objetivo de levantar en la producción científica brasileña de enfermería los cuidados de enfermería administrados al interno en situación de cárcel, se elaboró un $2^{\circ}$ cuadro, que tuvo por finalidad reunir los títulos de las publicaciones, seguido de los objetivos de los trabajos, para favorecer el destaque de las categorías que emergiesen tras esta clasificación. 


\begin{tabular}{|c|c|}
\hline Título & Objetivo \\
\hline $\begin{array}{l}\text { Cuidar de Apenados: percepção da equipe } \\
\text { de enfermagem. }\end{array}$ & $\begin{array}{l}\text { Conocer las percepciones y sentimientos del } \\
\text { equipo de enfermería al cuidar de penados en } \\
\text { el ámbito hospitalario; }\end{array}$ \\
\hline $\begin{array}{l}\text { Nascimento atrás das grades: uma pratica } \\
\text { de cuidado direcionado a gestantes, } \\
\text { puérperas e recém-nascidos em privação } \\
\text { de liberdade }\end{array}$ & $\begin{array}{l}\text { Desarrollar una práctica de cuidado dirigida a } \\
\text { gestantes, puérperas y recién nacidos en } \\
\text { privación de libertad, orientada por la Teoría } \\
\text { General de Enfermería de Orem; }\end{array}$ \\
\hline $\begin{array}{l}\text { As Marcas da Dupla Exclusão: } \\
\text { Experiências da Enfermagem com o } \\
\text { Psicótico Infrator. }\end{array}$ & $\begin{array}{l}\text { Describir las actitudes de los profesionales de } \\
\text { enfermería en el cuidado al psicótico } \\
\text { infractor, identificando las consecuencias de } \\
\text { esas actitudes en el cuidado de enfermería; }\end{array}$ \\
\hline $\begin{array}{l}\text { A Prática de Enfermagem no Sistema } \\
\text { Penal: Limites e Possibilidades. }\end{array}$ & $\begin{array}{l}\text { Identificar los princípios que señalan la } \\
\text { práctica de enfermería y discutir los límites y } \\
\text { las posibilidades de actuación del equipo de } \\
\text { enfermería en los servicios de salud del } \\
\text { Sistema Penal de RJ; }\end{array}$ \\
\hline
\end{tabular}

El análisis cualitativo de informaciones contó con las prerrogativas del análisis de contenido, esto solamente ocurre a partir de la comprensión del contenido de las ideas de los autores, pues a través del análisis de contenido podemos confirmar o no las informaciones establecidas antes del trabajo de investigación ${ }^{(15)}$.

En esta fase del trabajo se contó también con la contribución de Waldow ${ }^{2}$ para clasificar los cuidados de enfermería, identificados en los textos, pues para la autora el cuidado humano se estructura en dos ejes de significancia: El Cuidado Expresivo y el Cuidado Profesional.

$\checkmark$ Cuidado expresivo: es el que involucra acciones educativas, apoyo emocional, consuelo espiritual y expresión de sentimentos;

$\checkmark$ Cuidado profesional: es el que involucra competencia técnica y conocimiento profesional, para asistir y cuidar al paciente como un todo.

\section{DISCUSIÓN Y ANÁLISIS DE LAS INFORMACIONES}

La lectura analítica realizada sobre todo el material pertinente dirigió el análisis cualitativo de informaciones, solamente alcanzado tras la comprensión del contenido de las ideas de los autores, lo que permitió la elaboración de 03 categorías de desarrollo del trabajo, sobre los cuidados de Enfermería promovidos en Situación de Cárcel. 


\section{Categoría 1:}

\section{Cuidados de Enfermería: la ética profesional entre las limitaciones de un sistema.}

Esta categoría temática objetivó levantar los cuidados de enfermería a partir del estudio realizado en dos artículos científicos que son: Cuidar de penados: percepción del equipo de enfermería y La Práctica de Enfermería en el Sistema Penal: Límites y Posibilidades, que tuvieron como escenario el CTI de un Hospital General de gran porte en Rio Grande do Sul y un Hospital General del Sistema Penal de Rio de Janeiro.

La enfermería es un proceso de acción, reacción, interacción y transación entre individuos y grupos en un sistema social, cuya acción se desarrolla en determinado ambiente. El ambiente trae consigo la concienciación de los porqués y para qué nos encontramos en un lugar, haciendo o recibiendo algo de alguien, en el caso de la enfermería, la meta es actuar profesionalmente, para alcanzar de forma sistemática los objetivos de salud o ajuste de los problemas, para ello, asociados a la técnica, son utilizados los recursos mentales de percepción, para orientar al profesional sobre posibles señales y síntomas provocados por la patología ${ }^{(16)}$.

El ambiente donde ocurren las acciones para el cuidado tiene gran influencia en las relaciones, pues la forma como el sujeto aprende y comprende el ambiente como contexto humano de relaciones, y se percibe en el mismo, puede influenciar tanto favoreciendo como dificultando la integración y el crecimiento de los sujetos involucrados en la relación. En el caso de las unidades privadoras de la libertad humana, como ambiente de cuidados, esa relación tal vez sea aún más compleja, pues estos lugares son la parte oscura, que nos negamos a ver, la otra cara de la moneda ${ }^{(16)}$.

En el ejercicio profesional, la enfermería se depara con situaciones limitrofes entre el cuidar y cómo cuidar, pues de forma inconsciente, los valores morales adquiridos como ser humano, en algún momento, pueden entrar en conflicto con los valores dictados por la profesión. Aunque para los equipos de enfermería que desempeñan sus funciones en hospitales generales de la red pública de salud, el cuidar de infractores en enfermerías y la convivencia con los armamentos pesados del equipo de seguridad y custodia, en un pasado próximo, forman parte de la realidad y la rutina de los profesionales. Siendo el más reciente para la categoría, actualmente, la actuación del equipo de enfermería en los cuidados, intramuros institucional ${ }^{(17)}$.

En los presidios, profesionales y pacientes comparten sentimientos que pueden favorecer o perjudicar el cuidado, entre estos sentimientos, el miedo de los profesionales durante la atención a los presos, que temiendo por la integridad física, se hace a veces intenso, hasta incluso por ser algo propio del ser humano, que se liga al instinto de preservación, y acontece independientemente del querer, que ocurre como garantía contra el peligro ${ }^{(17)}$. Una u otra cuestión no se pueden dejar de lado, teniendo en cuenta los diversos riesgos a los que estos profesionales están expuestos, como biológicos, químicos, ergonómicos entre otros citados anteriormente. Se suma a estos el bregar con lo desconocido, o adaptarse a lo nuevo sin un soporte efectivo y continuo que garantice la seguridad de profesionales y pacientes ${ }^{(18)}$. 
Las acciones inherentes a la profesión de enfermería, sean administrativas o asistenciales, se diferencian en su aplicabilidad si las comparamos con las realizadas extramuros del Sistema Penal ${ }^{(16)}$. Tal vez porque estas acciones están sometidas a limitación espacial y por la presencia constante del agente penitenciario, cuya permanencia es obligatoria en cualquier procedimiento, y debido a la sensación de vulnerabilidad, producida por el miedo, la presencia del equipo de seguridad es entendida como una necesidad para la administración de cuidados técnicos ${ }^{(17)}$.

El estudio de esta categoría contribuyó a la percepción de que la enfermería presenta algunos sesgos ético-morales relacionados con la administración de cuidados. Ambos sesgos sobrepasan las limitaciones de un sistema, cuyo estigma de violencia estimula el miedo en el equipo de enfermería que, atendiendo a la ética profesional ${ }^{(19)}$, administra cuidados de forma técnica, rodeados por una serie de otras limitaciones como la espacial, debido al espacio reducido de las celdas; por la disponibilidad del equipo de seguridad -una limitación a la espontaneidad e interacción de la enfermería, en el desarrollo de la asistencia, y al mismo tiempo, una necesdad para la seguridad del equipo, a partir de su presencia constante.

Es sabido que no todos los presos constituyen amenazas inminentes, por eso en el grupo, existe también la dificultad de identificarse entre uno y otro, hecho que corrobora el que el equipo de enfermería, constantemente se mantenga en estado de defensa y alerta. Así atendiendo al objetivo de este estudio, esta categoría resaltó los cuidados de enfermería, administrados de forma profesional, conforme a la clasificación de Waldow ${ }^{(2)}$.

\section{Categoría 2:}

\section{Experiencia educacional entre barreras.}

El cuidado de enfermería como proceso educativo y de inclusión social abordado en esta categoría, apuntó varias vertientes, tal vez por haber resultado la investigación en estudios con foco en escenarios de diferentes características, para clientela también diferenciada.

Aunque realizado un estudio ${ }^{(20)}$ en una unidad de salud del Sistema Penal se dio en una unidad carcelaria femenina común, y buscó analizar el universo de algunas mujeres que en algún momento de sus vidas, incluso internas, vivenciaron también el proceso reproductivo, lo que objetivó el desarrollo de un proyecto asistencial a las gestantes, a las puérperas y a los recién nacidos.

La Constitución Federal de Brasil de 1988, a través de la Ley no 7210 , prevé el derecho de las presidiarias a estar con sus bebés durante el período de amamantación ${ }^{(20)}$. La gestación es un periodo en que la historia que cada mujer trae, debe ser acogida integralmente a partir del relato de la gestante y de sus acompañantes. Son también parte de esa historia los hechos, emociones o sentimientos, percibidos por los miembros del equipo involucrado en el pre-natal ${ }^{(21)}$.

Basándose en estas premisas y para dar sustento teórico al proceso de enfermería junto a esta población, las autoras utilizaron la Teoría del Autocuidado de Orem en el referido estudio ${ }^{(20)}$. La educación para el autocuidado es un proceso dinámico que depende de la voluntad del cliente y de la percepción que este tiene sobre su condición clínica. En ella los pacientes juzgan si la acción de autocuidado es benéfica, 
y ese juicio ocurre de acuerdo con las orientaciones internas y/o externas que, a su vez, son moldeadas por la cultura en que los individuos viven ${ }^{(22)}$.

Este es un abordaje que comprende los requisitos de autocuidado como universales y básicos para el mantenimiento de la vida; de desarrollo, relativos a períodos específicos del ciclo vital; y de cambios de salud, asociados con alteraciones que pueden ocurrir durante el curso del ciclo vital ${ }^{(22)}$.

La mayoría de las intervenciones en enfermería son acciones de orden educativo, que siguen el curso de las orientaciones, y en cierta forma pueden invadir la privacidad individual o cultural de las personas y, para que se consiga la aquiescencia del paciente/cliente, es necesario que el profesional de enfermería, además de buscar familiarizarse con la situación clínica y cognitiva del paciente venga también a utilizar algún recurso extra para sensibilizar y despertar su atención, atendiendo a la continuidad de los cuidados. En este objetivo la acción del educador no es solamente transferir conocimientos, sino crear alternativas para que ellos ocurran ${ }^{(23)}$.

La dinámica de abordaje se dio a partir del proceso de enfermería: colecta de datos, a partir de la consulta de enfermería, establecimiento de los diagnósticos de enfermería provenientes de las demandas entre la exigencia del autocuidado y la habilidad de las mujeres para su autocuidado, planificación e implementación del cuidado propiamente dicho y la evolución de enfermería, considerada como momento de validación del plan y de las acciones ${ }^{(20)}$.

Una buena forma de conocer el mundo sería someterse a la compañía de sus participantes, de acuerdo con las pequeñas coyunturas a que están sujetos ${ }^{(10)}$. En ese sentido la estrategia utilizada fue la aproximación y la interacción del equipo de enfermería con las internas, a partir del abordaje colectivo y de oficinas educativas, de forma que se prioricen los momentos individuales entre madre e hijo, intercalados con actividades educativas en grupo, que demostraron ser fundamentales para el proyecto de desarrollo de los cuidados de enfermería integral y complementar a este grupo de mujeres $^{(20)}$. La característica femenina, que por su biología permite la continuidad de la vida, también se mostró fértil en recibir y en reproducir los cuidados, aprendidos de las acciones educativas de enfermería, pues el cuidar en cualquier área de la salud, en especial en la obstetricia, no es un cuidar depositario de informaciones, por el contrario, es dinámico e interactúa todo el tiempo con el estimulador.

El cuidado de enfermería descrito en esta categoría, sobrepasó el proceso educativo, pues las internas al incluirse como promotoras del propio autocuidado y de cuidados como la higiene y amamantanamiento de los hijos recién nacidos, promovieron también un reencuentro con estructuras socioafectivas: como sus humanidades condición especial de actuar, sentir y existir como ser humano, y con el futuro, representado por los bebés, una parte de la sociedad, que irá creciendo y desarrollándose y que tuvo inicio allí, en la cárcel y en vientres encarcelados, lo que denota que lo aprendido sobre el amor, no se deja aprisionar por muros altos o por barreras, para mostrarse en esencia. Por otro lado la experiencia trajo intramuros institucional, la presencia de otros profesionales de salud, a través de las acciones de promoción de la salud, como la inmunización y proyección neonatal, lo que se acredita haber contribuido a que se desmitificasen posibles distorsiones en cuanto al ambiente o la clientela atendida ${ }^{(20)}$. 
Desde el punto de vista de la enfermería, a partir del abordaje educativo en la cárcel, resulta la posibilidad de cambio de paradigmas, como la apertura de un nuevo campo de estudio y de actuación profesional, ocasión que permitió la igualdad de acceso de las poblaciones más carentes, conforme los principios de igualdad, universalidad y equidad que guían el SUS ${ }^{(6)}$.

En esta categoría se identificaron cuidados de enfermería, que para ser administrados, contaron con la aquiescencia de las internas en desarrollar actividades educativas, de apoyo emocional, consuelo espiritual y expresión de sentimientos, como las descritas por Waldow ${ }^{(2)}$, en las acciones de cuidados expresivos.

\section{Categoría 3:}

\section{Ambigüedad: los cuidados de enfermería entre el custodiar y el tratar}

Esta categoría objetiva abordar las vicisitudes comportamentales existentes entre el equipo de enfermería y el psicótico infractor, así como busca identificar los cuidados de enfermería dispensados a esta población. El escenario fue un Hospital de Custodia y Tratamiento Psiquiátrico en RJ. El loco es aquel que tiene deficiencia de las facultades mentales, está incapacitado para comprender lo que está bien o mal. Lidiar con el portador de enfermedad mental, siempre fue un desafío para la sociedad. El loco-criminal actúa de forma diferente del loco común, es un individuo incapaz de contener los impulsos criminales derivados de su anomalía psíquica ${ }^{(11)}$.

La categoría de criminal y al mismo tiempo portador de trastorno mental estrecha las relaciones entre la medicina psiquiátrica y la Justicia penal, ya que la Justicia solamente puede actuar tras la ejecución de un delito; por otro lado, la psiquiatría reune condiciones de preverlo en función de criterios de peligrosidad, definidos científicamente, de ahí la denominación clínicamente adoptada de calificar al lococriminal de psicótico-infractor ${ }^{(11)}$.

Declarar alguien, al mismo tiempo culpable y loco retira de la Justicia el poder sobre el autor del delito, con todo para defender la sociedad de la peligrosidad de un psicóticoinfractor, surge la medida de seguridad, conciliadora entre Justicia y Medicina, como un recurso que tiende a proporcionar al loco-criminal, un destino diferenciado del criminal común. Vale resaltar que las medidas de seguridad a su alcance preven perspectivas preventivas y terapéuticas, no siendo considerada como acto punitivo, pues prescribe tratamiento de salud mental del individuo, a pesar de ser cumplida también en régimen cerrado ${ }^{(11)}$.

El equipo multiprofesional de salud, del manicomio judicial, sin una rutina establecida, actúa individualmente, enalteciendo al equipo de seguridad, para crear un ambiente terapéutico, que estimule a los internos a participar de oficinas de arcilla y tierra, sesiones de musicoterapia, de charlas y debates así como de salidas terapéuticas, casi siempre vetadas, sin causas aparentes. En ese escenario, los equipos de enfermería desarrollan sus actividades en los HCTPs, buscando entender su papel, y siguen administrando cuidados profesionales, que se mueven entre conflictos éticomorales, pues siendo el único grupo del equipo multiprofesional que pasa 24 horas seguidas en contacto con los internos, están más expuestos a la masificación de informaciones sobre la historia precedente del interno y de su grado de peligrosidad, tendiendo entonces, a reproducir actos y acciones, oriundas del pasado, como el 
establecimiento de la relación vertical de poder y de vigilancia, reduciendo al paciente a la condición de sumisión y de obediencia ${ }^{(11)}$.

Esta categoría contribuyó a destacar la ambigüedad de un servicio que al mismo tiempo que busca tratar al paciente con los recursos multidisciplinares y multiprofesionales, encuentra en el equipo de seguridad, el start que liga el botón de la concienciación para la real vocación del lugar, juntamente con sus estigmas y peligros. Y en ese ambiente de tratar y custodiar, los contrastes se contraponen en la mente de las personas de tal forma, que la asistencia al interno queda de modo general, sometida a la definición o norte de la acción individual adoptada por cada profesional, lo que corrobora que la asistencia de enfermería está influida por esta corriente y administra cuidados profesionales y de cuño moral, pasados al paciente de forma mecanizada.

El estudio también apuntó la necesidad de revisiones en el papel y en la conducta de los profesionales de enfermería en el desarrollo de cuidados al infractor psicótico, que tal vez a partir de un plan de cuidados específicos, pueda contribuir a una asistencia de enfermería cristalina, sin las influencias individuales. La línea de cuidados identificados en esta categoría se encuadra según estudios de Waldow ${ }^{(2)}$, como cuidados de enfermería administrados de forma profesional.

\section{CONSIDERACIONES FINALES}

La presente revisión de literatura contó con cuatro textos que subsidiaron la elaboración de tres categorías de desarrollo, clasificadas conforme los conceptos teóricos de Waldow ${ }^{2}$, sobre el Cuidado Profesional y el Cuidado Expresivo. El análisis de las informaciones apuntó el predominio en $75 \%$ del Cuidado Profesional y $25 \%$ referido al Cuidado Expresivo, como acciones de enfermería.

En relación al objetivo propuesto por esta revisión, se verificó que la práctica del cuidado de enfermería en situación de cárcel se sitúa entre dos polos: el primero se refiere a una visión positiva en cuanto a los derechos del tutelado, visto que el hecho de encontrarse cumpliendo pena en la Unidad Prisional o medida de seguridad, no le retira la prerrogativa de ciudadano de tener sus derechos preservados, configurando el Cuidado Profesional y el Expresivo, como acciones que se establecen en la relación de la ética humana y profesional. Por tanto en este caso, el cuidar parece dejar de ser solamente un procedimiento, una intervención, para ser una relación, donde la ayuda es humanitaria, y el objetivo es el de invertir en la cualidad del otro ser o venir a ser, respetándolo, comprendiéndolo, tocándolo de forma más afectiva.

El otro polo destacado se refiere a los preconceptos y dilemas morales, que están directamente arraigados entre sí, que refieren el miedo como un derivado de la visión que se tiene de los presos y de los locos, pues la sociedad los ve como alguien capaz de hacer el mal a quien quiera que sea; y la interferencia causada por el sistema físico-humano de seguridad, visto que al mismo tiempo en que protege, es capaz de avivar en el interior de las personas el resquicio del miedo social, cuyo cansancio físico-mental, debido al tiempo de exposición al ambiente, tiende a ampliar exponencialmente, en el equipo de enfermería, no sólo la sensación de miedo, sino cualquier otra sensación negativa.

Otra cuestión que no puede dejarse de lado es que, teniendo en cuenta los diversos riesgos a los que estos profesionales están expuestos, como biológicos, químicos, 
ergonómicos entre otros citados anteriormente, se suma a estos el lidiar con lo desconocido, el adaptarse a lo nuevo sin un soporte efectivo y continuo que garantice la seguridad de profesionales y pacientes. Con todo, el miedo se muestra de forma generalizada, explicado por la propia naturaleza humana, de preservación de la vida, cuando percibe algún tipo de riesgo, ampliado por un ambiente inhóspito, donde todos los actores, internos y/o profesionaless, son desconocidos y, potencialmente, pueden representar de alguna forma amenaza a la integridad física. Así sienten miedo, los profesionales de enfermería, de la seguridad y de los propios internos.

Al utilizar las funciones físico mentales para estimular al paciente, lo que la enfermería busca es hacer que el sujeto de cuidados se sienta apoyado y hasta incluso valorizado y sus necesidades, y la meta es contribuir a mejorar las condiciones de vida de las personas, a través del estímulo de la autoestima.

La administración tanto del Cuidado Expresivo como del Cuidado Profesional necesita de la evaluación del (a) enfermero (a), para surtir los efectos deseados, pues la elección incorrecta del abordaje puede desarrollar en el paciente el sentimiento de dependencia y de incapacidad para realizar sus propias acciones y/o de lo contrario, no surtir el efecto deseado, debido muchas veces a la incapacidad de entendimiento del paciente en realizarlas.

\section{REFERENCIAS}

1- Boff L. Saber Cuidar: ética do humano - compaixão pela terra. 9ạed. Rio de Janeiro (RJ). Vozes, 1999.

2- Waldow,VR. Cuidado humano: o resgate necessário. Porto Alegre: Sagra; 1999.

3- Toledo, MM; Soares TC. A percepção dos Enfermeiros sobre sua Prática: a ótica do profissional no contexto hospitalar. Nursing (São Paulo). 2008;11(120):214-218.

4- Zoboli PLC. A Redescoberta da Ética do Cuidado: o foco e a ênfase nas relações. Rev. Esc. Enferm. USP. 2004; 38(1): 21-27.

5-Cintra EA. et al. Assistência de Enfermagem ao Paciente Crítico. São Paulo: Atheneu.2000.

6- Ministério da Saúde(Br). Lei 8080 de 19 de março de 1984. Dispõe sobre as condições para promoção, proteção e recuperação da saúde, a organização e o funcionamento dos serviços correspondentes e dá outras providências. Brasília. DF. 2000.

7- Ministério da Saúde(Br). Plano Nacional de Saúde no Sistema Penitenciário. Portaria Interministerial no 1.777 de 09 de novembro de 2003. Brasília, DF. 2004.

8- Ministério da Justiça(Br). Lei 2.848 de 07 de dezembro de 1940. Dispõe sobre o Código Penal Brasileiro. São Paulo; Saraiva 2002.

9- Mirabete F. Execução Penal. 9ª ed. São Paulo: Atlas; 2000.

10- Goffman, I. Manicômios, prisões e conventos. $7^{\mathfrak{a}}$ ed. São Paulo (SP): Perspectiva; 2001.

11- Santos, MLSC. Souza, SF, Santos, CVSC. As Marcas da Dupla Exclusão: experiências da enfermagem com o psicótico infrator. Texto e Contexto Enferm,Florianópolis,2006, 15 (Esp): 79872006.

12- Gil, AC. Como elaborar projeto de pesquisa. São Paulo: Atlas, 2002.

13- Marconi, MA. Lakatos, EM. Técnicas de Pesquisa. 5ee. São Paulo(SP): Atlas, 2002.

14- Cervo, AL.; Bervian, PA. Metodologia Científica. 5 ed. São Paulo(SP): Pearson Prentice Hall, 2005. 
15- Minayo, MCS. Pesquisa Social: teoria, método e criatividade. 11 $11^{\text {a }}$ ed. Petrópolis: Vozes; 2000.

16- Souza MOS. Passos, JP. A prática de Enfermagem no Sistema Penal: limites e possibilidades. Esc. Anna Nery. Rev. Enferm. 2008. 12(3): 417-23.

17- Rosa SRF. Cuidar de Apenados: percepção da equipe de enfermagem. Nursing (São Paulo). 2004; 7(75): 27-35.

18- Valente, GSC. Sampaio, SZ. Fernandes, DMM. a Saúde do Trabalhador de Enfermagem: implicações a partir da oficialização da NR_32. Revista de enfermagem UFPE. 2011;5(9):2105-110.

19- Conselho Federal de Enfermagem (Br). Resolução COFEN no 240 de 30 de agosto de 2000: dispõe sobre os princípios fundamentais, aprova o código de ética dos profissionais de enfermagem e dá outras providências[internet].Disponível em: http://www.portalcofen.gov.br/2010/matérias.

20- Rosinski,TC.Cordeiro,CG. Monticelli,M. Santos,EKG. Nascimento Atrás das Grades: uma prática direcionada à gestantes, puérperas e recém-nascidos em privação de liberdade. Rev. Ciência, Cuidado e Saúde. 2006 5(2); 211-219. 